\title{
Reduced-Order Modeling of Reacting Supersonic Flows in Scramjet Nozzles
}

\author{
Derek J. Dalle, Sean M. Torrez, James F. Driscoll ${ }^{\dagger}$ \\ University of Michigan, Ann Arbor, MI 48109
}

\begin{abstract}
Control-oriented models of hypersonic vehicle propulsion systems require a reduced-order model of the scramjet nozzle that is accurate to within $10 \%$ but requires less than a few seconds of computational time. To achieve this goal, a reduced-order model is presented, which predicts the solution of a steady two-dimensional supersonic flow through a nozzle or around any other two-dimensional geometry. Expansion fans are modeled as a sequence of discrete waves instead of a continuous pressure change. Of critical importance to the model is the ability to predict the results of multiple wave interactions rapidly. A reduced mechanism is used to account for finite-rate chemistry, which is needed to accurately account for recombination in the nozzle. To assess the accuracy of the proposed method, some comparisons to CFD solutions of nozzle flows are presented.
\end{abstract}

\section{Nomenclature}

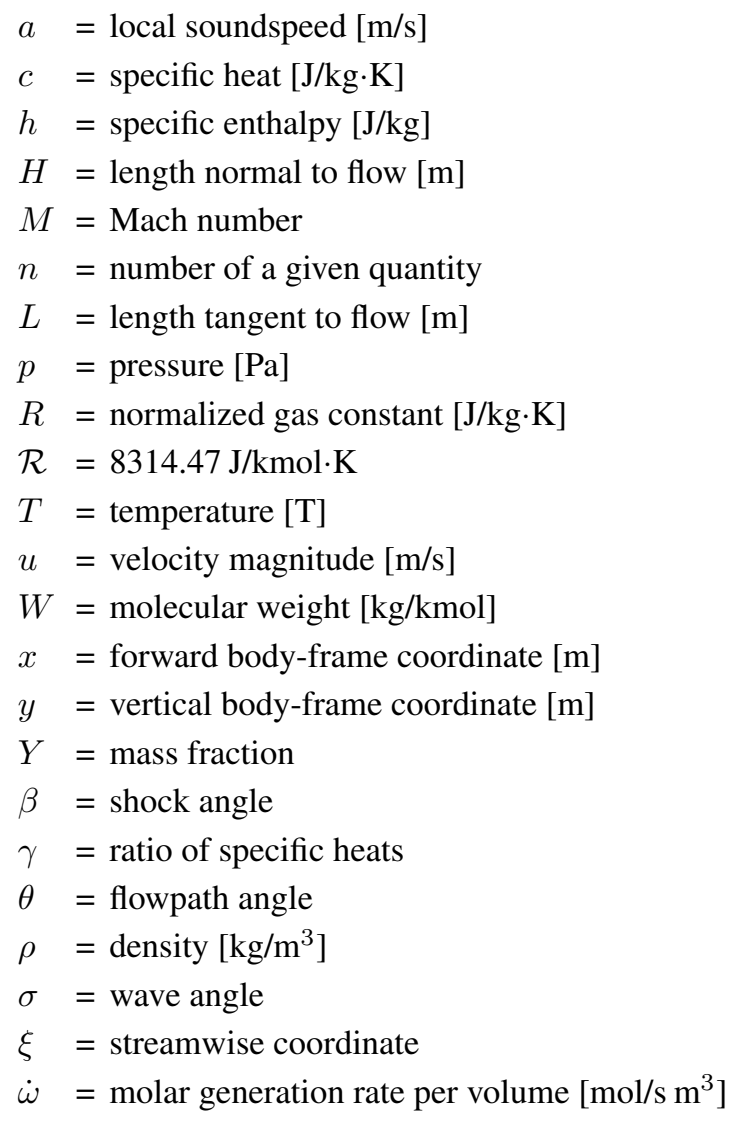

* Graduate Research Assistant, Department of Aerospace Engineering, AIAA Student Member

${ }^{\dagger}$ Professor, Department of Aerospace Engineering, AIAA Fellow 


$\begin{array}{ll} & \text { subscripts } \\ i & \text { species index } \\ p & \text { constant pressure } \\ \text { sp } & \text { pertaining to species } \\ 0 & \text { stagnation value } \\ \infty & \text { freestream } \\ \text { superscripts } \\ * & \text { value at Mach number of } 1 \\ + & \text { reference value for boundary layer }\end{array}$

\section{Introduction}

The ability to estimate the properties of a supersonic flow quickly is critical for the design of a control-oriented model of a hypersonic vehicle. For example, a control algorithm must rapidly compute the thrust along a vehicle trajectory as the flight Mach number and angle of attack continuously change. The algorithm must also calculate the thrust for any perturbations to the design trajectory. Examples of simulations that require such a model include MASIV (Michigan/AFRL Scramjet In Vehicle) ${ }^{1-3}$ and work by Frendreis et. al. ${ }^{4}$

While simple tools such as Newtonian impact theory and piston theory can be used to estimate lift and drag, they are not appropriate on surfaces that are downstream of the vehicle such as those that make up the nozzle. Furthermore, estimating the performance of a dual-mode scramjet requires accurate information about the properties of the fluid flow as it leaves the inlet and enters the isolator. The use of high-fidelity CFD can be employed to determine the flow through the inlet accurately, but this solution requires too much computational time to be viable for control-oriented modeling. On the other hand, simple models have been used by Bolender and Doman, ${ }^{5}$ and Cubbage et. al. ${ }^{6}$ and others to estimate the performance of the nozzle. These models essentially model the nozzle as a one-dimensional expansion. While a one-dimensional model may be able to predict thrust relatively well in certainrocket nozzles, expansion in a scramjet nozzle is an inherently two-dimensional process. Unlike a traditional rocket nozzle, the scramjet nozzle has a lower boundary that is a free shear layer rather than a geometric wall. The location of the cruved free shear layer has a significan effect on engine thrus and requires two-dimensional modeling. There has also been a significant amount of work in high-fidelity CFD an experimental validation. ${ }^{7,8}$ Applying our wave-based aerodynamic model, ${ }^{9}$ which was developed for scramjet inlet analysis, is a natural extension of previous work and a compromise between the speed of a one-dimensional model and the accuracy of full CFD.

A satisfactory approach must require a relatively small amount of computational time and still yield a relatively accurate solution for the nozzle flow. Instead of solving directly for the flow conditions at each point in the flow (as is done in CFD), we propose a method that solves for the positions of the relevant waves directly. These waves separate regions in which the flow properties are uniform. The proposed method rapidly provides solutions for the locations of the shock waves and expansions using established two-dimensional supersonic theory. To make this possible in a digital computing environment, we approximate expansion fans as a number of discrete waves, and we model curved surfaces as a number of straight sections. After determining the locations at which two or more waves intersect, the program solves the interactions among the waves as two-dimensional Riemann problems. In many ways this is a generalized and automated version of the method of characteristics.

We introduce a reduced-order model for two-dimensional finite rate chemistry. Finite-rate chemistry is expected to play an important role in the nozzle ${ }^{10}$ for at least two reasons: recombination and unburned fuel. Recombining even a small mass of radicals can release a significant amount of heat. However, due to the very large kinetic energies present in hypersonic flow, the amount of heat released may not be enough to have a noticeable effect on the thrust. In the reduced-order chemistry model, we assume that chemistry occurs along each streamline independently of neighboring streamlines, which allows us to use a one-dimensional chemistry model for each streamline.

An example vehicle geometry, shown in Fig. 1, shows how the nozzle integrates with the other vehicle components. An example nozzle geometry is shown in Fig. 2 using the same station numbering as Fig. 1. A shock wave can be seen downstream of the cowl trailing edge in Fig. 2. The difference in pressure at the cowl trailing edge allows the exhaust plume to adapt to different conditions, and gives a similar performance to an aerospike nozzle. ${ }^{11-13}$

The most important feature of a reduced-order model is the ability to estimate the nozzle performance over a wide range of operating conditions. It is possible, using the method of characteristics, to design a two-dimensional nozzle that has a very simple exhaust pattern. However, if the exhaust pressure at the trailing edge is different from the 


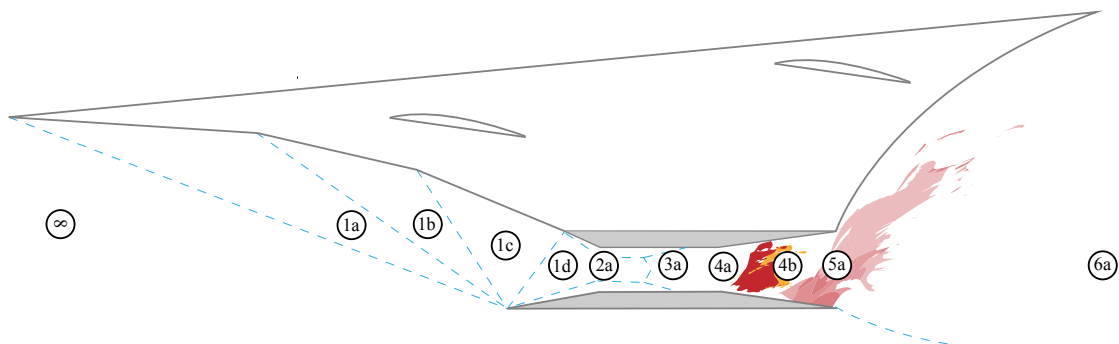

Figure 1. An example vehicle geometry with extensible station numbering.

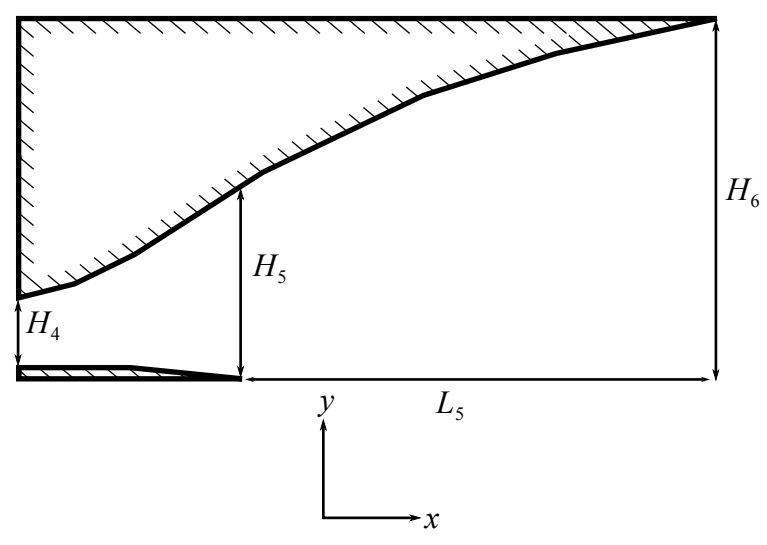

a) Nozzle geometry

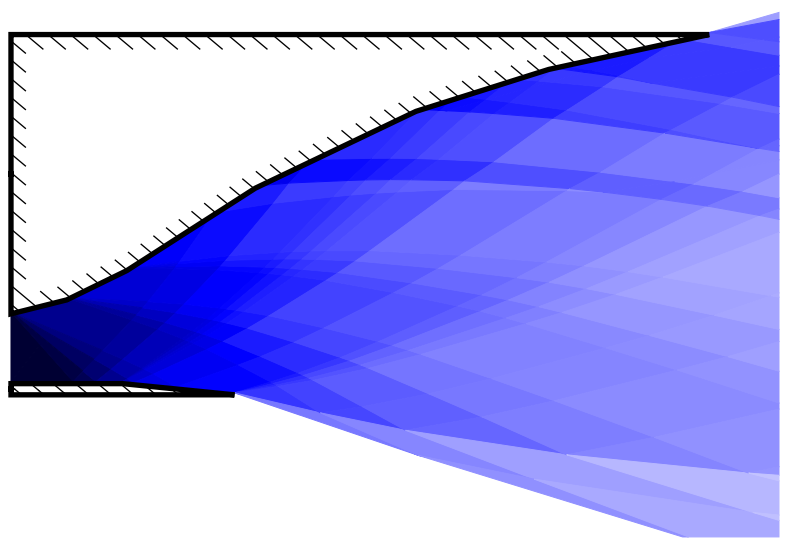

b) Reduced-order solution

Figure 2. A sketch of a potential nozzle geometry and a corresponding flow solution. Darker shades of blue represent regions of higher temperature.

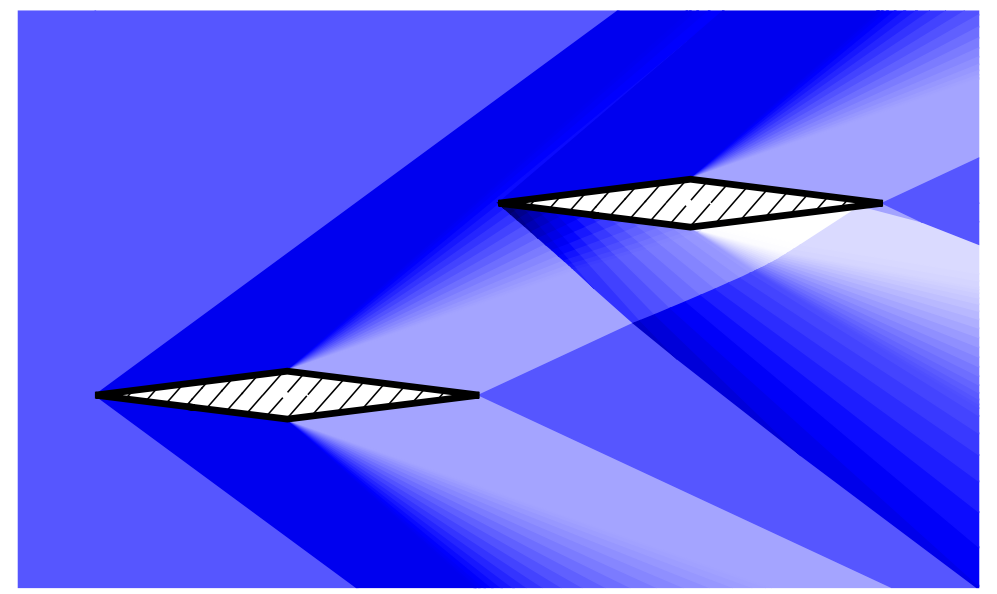

Figure 3. The inviscid flow over two diamond airfoils at an angle of attack of $\alpha=0$ and freestream Mach number of $M_{\infty}=2$ is shown. Darker shades of blue represent regions of higher pressure while white corresponds to the lowest pressure. 
freestream pressure, the method of characteristics cannot accurately predict the thrust due to the presence of shocks. As a result, a method that can model shocks, expansions, and interactions of the two is needed.

Although the analysis of scramjet inlets was the primary motivation to develop this solution method, it can be applied to any two-dimensional geometry for which there are no subsonic regions except for boundary layers and small subsonic regions in the near vicinity of blunted noses. An example that demonstrates the flexibility of the program is shown in Fig. 3. In this solution, the four expansion fans generated at vertices of the airfoils are modeled as twenty discrete waves.

\section{Finite-Rate Two-Dimensional Supersonic Flow Model}

Basic shock/expansion theory for two-dimensional calorically perfect gas can be found in many references. However, several modifications to the basic theory are made in our nozzle model. First, continuous expansion waves must be split into a finite number of discrete waves. We also incorporate finite-rate chemistry into our model. Finally, a method was devised to analyze wave interactions.

\section{A. Discretized Expansion Waves}

A schematic of a supersonic expansion is shown in Fig. 4. To analyze this situation, we must describe the thermodynamic variables in the post-expansion region, $\mathrm{B}$, and in the expansion region, between the waves labeled $\sigma_{\mathrm{A}}$ and $\sigma_{\mathrm{B}}$. Prandtl-Meyer theory is typically used to predict the flow properties of an expanding supersonic flow. However, in Prandtl-Meyer theory, the expansion region is a continuum of infinitesimal waves. Our algorithm requires discrete waves of finite strength. In addition, each wave must satisfy conservation of mass, momentum, and energy.

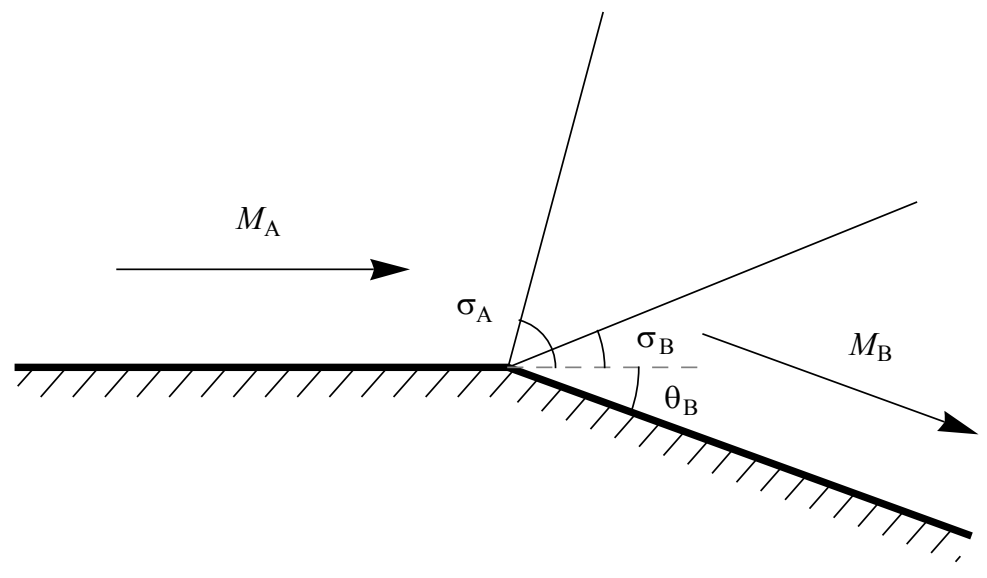

Figure 4. Illustration of a supersonic expansion wave

One approach is to discretize the Prandtl-Meyer expansion directly. This method, however, cannot satisfy conservation of mass and momentum. Figure 5 shows an example with the expansion modeled as a single wave. The control volume in Fig. 5 must not have any net flux of mass, $x$-momentum, or $y$-momentum. Since Prandtl-Meyer theory already predicts the thermodynamic quantities in the downstream state, the only degree of freedom in the model is the angle of the wave. There is no way to choose this one free variable to satisfy all three constraints. The only way to solve this problem is to allow the post-wave properties (state B) to vary slightly from the values predicted by Prandtl-Meyer. If we write the equations for conservation of mass, momentum, and energy for a discrete wave, we will obtain exactly the equations for an oblique shock. In other words, the best choice for a discrete wave is actually an expansion shock.

There are basically two possible choices. If the Prandtl-Meyer wave is discretized directly, the predicted pressure, density, etc. will be calculated exactly, but mass and momentum will not be conserved no matter how finely the expansion fan is modeled. Using expansion shocks means that mass and momentum are conserved, but state B will not be exact. In a real flow, expansions shocks cannot occur because they decrease entropy. However, a sequence of weak expansion shocks is a close model of an expansion fan, and the entropy in region B will only be slightly lower than that in region $\mathrm{A}$. 


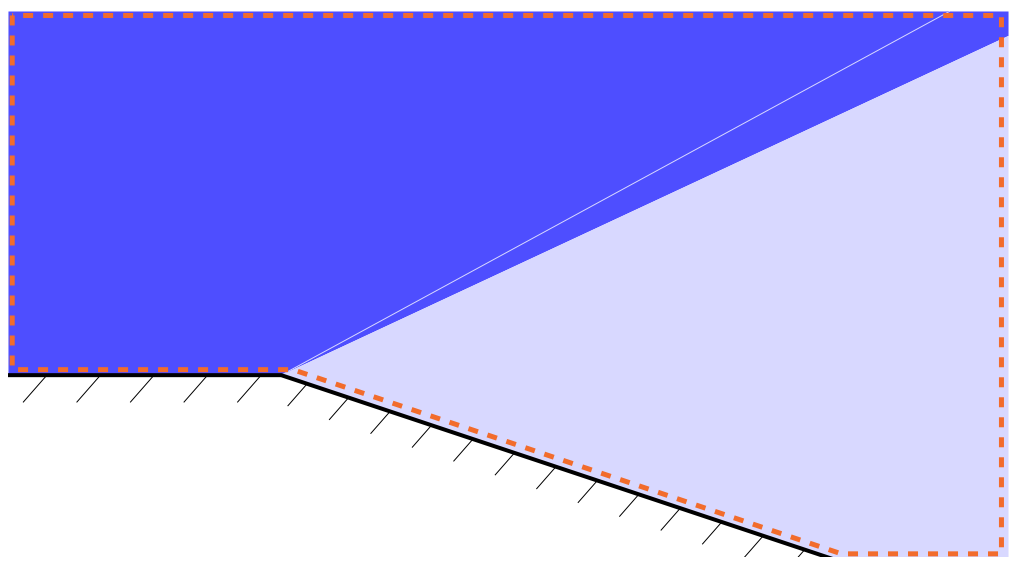

Figure 5. An expansion modeled as a single wave and a simple control volume.

If the goal is to predict lift and drag on the exterior part of a vehicle, the downstream pressure is the most important result, and using Prandtl-Meyer theory is often the best solution. However, thrust is proportional to the mass flow, so when analyzing a nozzle, expansion shocks are a better model. Since the proposed model will frequently use a control-volume type of analysis, conserving mass and momentum is critically important, and for this reason we use sequences of weak expansion shocks instead of Prandtl-Meyer theory.

The remaining task is to pick the sequence of expansion shocks that appropriately matches the expansion fan. We know that before the expansion, the flowpath angle is $\theta_{\mathrm{A}}$, and the flowpath angle is $\theta_{\mathrm{B}}$ after the expansion. Turning the expansion into $n_{\exp }$ discrete waves now reduces to picking a set of angles $\theta_{1}, \ldots, \theta_{n_{\exp }-1}$ to be the flowpath angles in the intermediate regions. Figure 6 shows an example with $n_{\exp }=4$. In this case, $\theta_{1}, \theta_{2}$, and $\theta_{3}$ are the flowpath angles in the three intermediate regions. There are many possible methods to select these intermediate angles, but we have opted to choose them based on the rules of Gaussian quadrature.

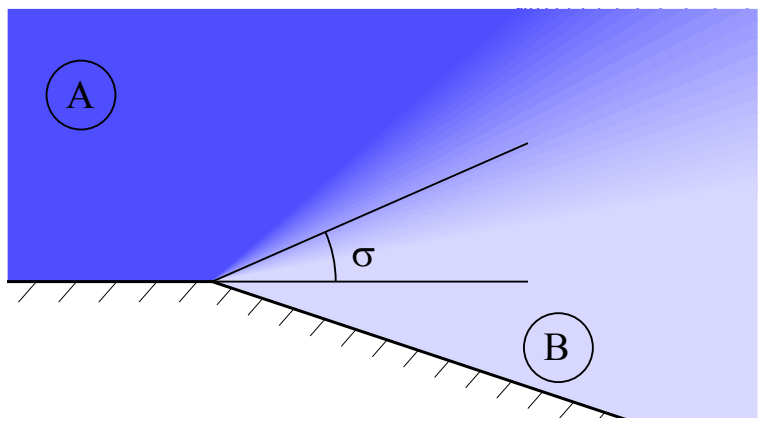

a) Smooth expansion

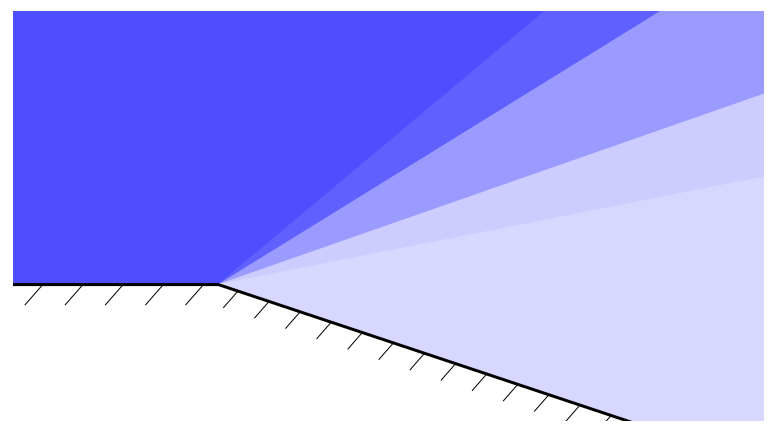

b) Discrete expansion with $n_{\exp }=4$

Figure 6. Two models of an expansion with $\theta_{\mathrm{B}}=-18.4^{\circ}$ and $M_{\infty}=1.5$

\section{B. One-Dimensional Chemistry Update}

Within each step, the thermodynamic state is updated using the assumption of constant-area, adiabatic, steady onedimensional flow. The equations of motion for this case can be reduced to a set of first-order ordinary differential equations. The dependent variable is the streamwise coordinate, $\xi$.

The first conservation equation is found by differentiating the equation of state, $p=\rho R T$, to get

$$
\frac{1}{p} \frac{d p}{d \xi}=\frac{1}{\rho} \frac{d \rho}{d \xi}+\frac{1}{R} \frac{d R}{d \xi}+\frac{1}{T} \frac{d T}{d \xi}
$$

Conservation of mass demands that $\rho u A$ is constant, which gives

$$
\frac{1}{\rho} \frac{d \rho}{d \xi}+\frac{1}{u} \frac{d u}{d \xi}+\frac{1}{A} \frac{d A}{d \xi}=0
$$


Conservation of momentum and energy take the form

$$
\begin{aligned}
\rho u \frac{d u}{d \xi} & =-\frac{d p}{d \xi} \\
\bar{c}_{p} \frac{d T}{d \xi} & =-u \frac{d u}{d \xi}-\sum_{i=1}^{n_{\mathrm{sp}}} h_{i} \frac{d Y_{i}}{d \xi}
\end{aligned}
$$

respectively, which comes from eliminating the time derivatives from the one-dimensional Navier-Stokes equations. Here $\bar{c}_{p}$ is the weight-averaged specific heat,

$$
\bar{c}_{p}=\sum_{i=1}^{n_{\mathrm{sp}}} c_{p, i} Y_{i}
$$

Finally, the chemistry takes the form

$$
u \frac{d Y_{i}}{d \xi}=\frac{W_{i}}{\rho} \dot{\omega}(p, T, \mathcal{Y})
$$

where $\dot{\omega}$ is the molar rate of production per unit volume.

The average molecular weight can be written

$$
W=\left(\sum_{i=1}^{n_{\mathrm{sp}}} \frac{Y_{i}}{W_{i}}\right)^{-1}
$$

so the spatial derivatives of the molecular weight and normalized gas constant are

$$
\begin{aligned}
\frac{d W}{d \xi} & =-W^{2} \sum_{i=1}^{n_{\mathrm{sp}}} \frac{1}{W_{i}} \frac{d Y_{i}}{d \xi} \\
\frac{d R}{d \xi} & =-\frac{R}{W} \frac{d W}{d \xi}
\end{aligned}
$$

respectively. The final step is to solve for one of the spatial derivatives, to make the solution straightforward. The velocity derivative is

$$
\frac{d u}{d \xi}=-\frac{p u}{\bar{c}_{p} T\left(p-\rho u^{2}\right)+p u^{2}}\left(\frac{\bar{c}_{p} T}{W} \frac{d W}{d \xi}+\frac{\bar{c}_{p} T}{A} \frac{d A}{d \xi}+\sum_{i=1}^{n_{\mathrm{sp}}} h_{i} \frac{d Y_{i}}{d \xi}\right)
$$

Since we assume $A$ is constant, one of the terms in Eq. 10 is zero. However, this term will be needed in the quasi-one-dimensional model.

The assumption of a one-dimensional constant-area flow is not exact for this case, because heat addition can cause one fluid element to expand relative to another one. However, this method does ensure that mass, momentum, and energy are conserved, and it will be accurate if the step size is adequately small.

\section{Reduced Chemistry Mechanism}

The current version of the code uses the reduced mechanism of Jachimowski, ${ }^{14}$ which uses 14 species (including Argon) and 33 one-way reactions. The mechanism has the capacity to model the combustion of hydrogen fuel, and it has been validated for high-temperature reactants. This mechanism gives the added capability that the code can analyze a nozzle in which there is unburned fuel leaving the combustor.

The enthalpies and specific heats are calculated using the NASA thermodynamic tables. ${ }^{15}$

\section{Two-dimensional Riemann Problem}

Consider the case of two interacting waves, which are shown on the left side of Fig. 7. The important parameters are the gas properties in regions A and D. The flow pattern on the right side of Fig. 7 is called the Riemann problem. It describes the situation in steady two-dimensional compressible flow in which two uniform regions are in contact with 


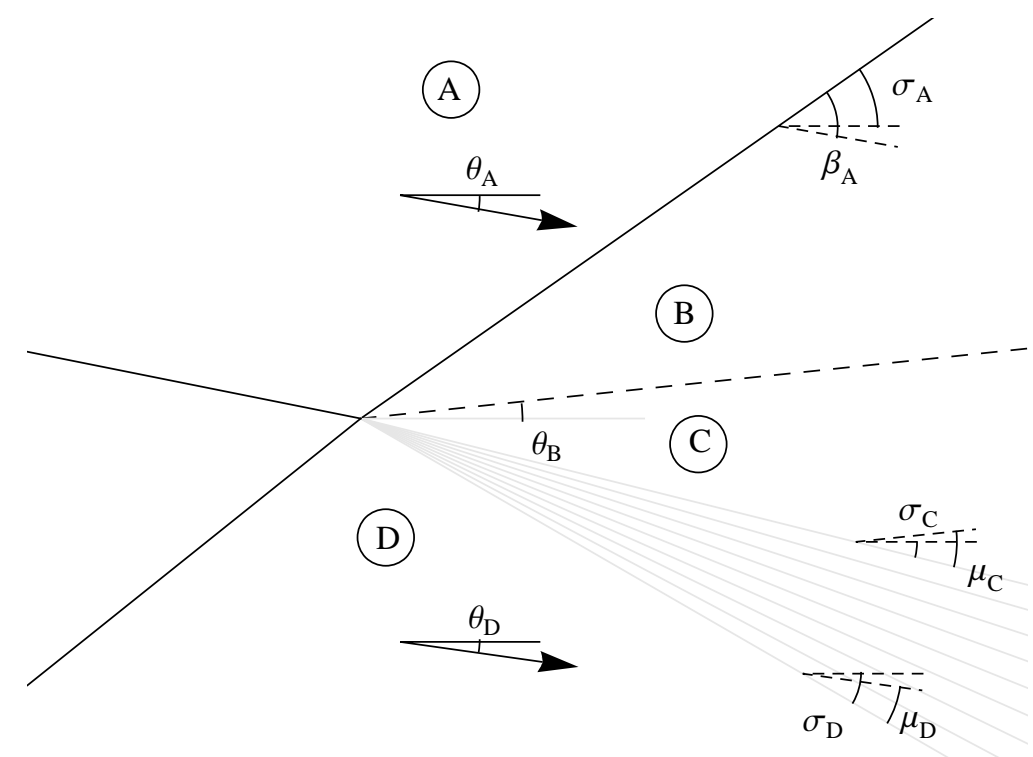

Figure 7. Geometry for a wave interaction with a shock and an expansion. In the general Riemann problem, both waves could be either a shock or an expansion. The combination of waves here indicates $p_{\mathrm{D}}>p_{\mathrm{A}}$.

each other. At the point of interaction, there are two inconsistent flow conditions. To rectify this situation, the gas in both regions must pass through a wave, as shown in Fig. 7.

The physical solution must have the property that the regions that are not separated by a shock or expansion must have equal pressures and flow in the same direction. Thus

$$
p_{\mathrm{B}}=p_{\mathrm{C}} \quad \text { and } \quad \theta_{\mathrm{B}}=\theta_{\mathrm{C}} .
$$

A simple, though inefficient, solution method is to construct a single pressure-deflection function

$$
p_{\mathrm{B}}=p\left(\mathrm{~A}, \theta_{\mathrm{B}}-\theta_{\mathrm{A}}\right)
$$

which takes as input all information about state $\mathrm{A}$ and a deflection angle $\theta_{\mathrm{B}}-\theta_{\mathrm{A}}$, and returns a post-wave pressure as output. The same function can be used to determine $p_{\mathrm{C}}$ using the formula

$$
p_{\mathrm{C}}=p\left(\mathrm{D}, \theta_{\mathrm{D}}-\theta_{\mathrm{C}}\right) .
$$

Opposite signs are used for the lower shock because the wave separating regions C and D is of the opposite family as the wave separating regions $\mathrm{A}$ and $\mathrm{B}$. Combining this pressure-deflection function with the constraints of equation (11) gives an equation

$$
p\left(\mathrm{~A}, \theta_{\mathrm{B}}-\theta_{\mathrm{A}}\right)=p\left(\mathrm{D}, \theta_{\mathrm{D}}-\theta_{\mathrm{B}}\right)
$$

which is satisfied only by the correct value of $\theta_{\mathrm{B}}$.

In order to reduce the number of waves present in a flow solution, it is advantageous to ignore waves that cause a negligible change in conditions. For example, if $T_{\mathrm{B}} / T_{\mathrm{A}}$ is very close to unity, the wave separating regions $\mathrm{A}$ and $\mathrm{B}$ plays a very minor role. In the proposed method, therefore, any of the three waves resulting from a wave interaction is ignored if the temperature jump across it is below a certain tolerance.

For an oblique shock, the pressure-deflection function is described by

$$
\begin{aligned}
& \frac{p_{\mathrm{B}}}{p_{\mathrm{A}}}=\frac{2 \gamma}{\gamma-1} M_{\mathrm{A}}^{2} \sin ^{2} \beta_{\mathrm{A}}^{2}-\frac{\gamma+1}{\gamma-1} \\
& \frac{p_{\mathrm{C}}}{p_{\mathrm{D}}}=\frac{2 \gamma}{\gamma-1} M_{l} \mathrm{~A}^{2} \sin ^{2} \beta_{\mathrm{D}}^{2}-\frac{\gamma+1}{\gamma-1}
\end{aligned}
$$


with the shock angles given by

$$
\begin{aligned}
& \tan \left(\theta_{\mathrm{B}}-\theta_{\mathrm{A}}\right)=C\left(M_{\mathrm{A}}, \beta_{\mathrm{A}}\right)=2 \cot \beta_{\mathrm{A}} \frac{M_{\mathrm{A}}^{2} \sin ^{2} \beta_{\mathrm{A}}-1}{M^{2}\left(\gamma+\cos 2 \beta_{\mathrm{A}}\right)+2} \\
& \tan \left(\theta_{\mathrm{D}}-\theta_{\mathrm{C}}\right)=C\left(M_{\mathrm{D}}, \beta_{\mathrm{D}}\right)=2 \cot \beta_{\mathrm{D}} \frac{M_{\mathrm{D}}^{2} \sin ^{2} \beta_{\mathrm{D}}-1}{M^{2}\left(\gamma+\cos 2 \beta_{\mathrm{D}}\right)+2}
\end{aligned}
$$

Combining Eqs. (11), (15), and (16) gives

$$
\beta_{\mathrm{D}}\left(\beta_{\mathrm{A}}\right)=\sin ^{-1} \sqrt{\frac{1}{M_{\mathrm{D}}^{2}}\left(\frac{p_{\mathrm{A}}}{p_{\mathrm{D}}}\left(M_{\mathrm{A}}^{2} \sin ^{2} \beta_{\mathrm{A}}-\frac{\gamma-1}{2 \gamma}\right)+\frac{\gamma-1}{2 \gamma}\right)}
$$

Substituting this result into Eqs. (17) and (18) and using the constraint $\theta_{\mathrm{B}}=\theta_{\mathrm{C}}$ gives an equation

$$
\theta_{\mathrm{A}}-\theta_{\mathrm{D}}+\tan ^{-1} C\left(\beta_{\mathrm{A}}, M_{\mathrm{A}}\right)+\tan ^{-1} C\left(\beta_{\mathrm{D}}\left(\beta_{\mathrm{A}}\right), M_{\mathrm{D}}\right)=0
$$

that is only satisfied for the correct value of $\beta_{\mathrm{A}}$.

\section{Proposed Model Supersonic Flow Discretization}

The proposed ROM (reduced-order model) is primarily concerned with the lines that separate regions of the flow that are considered to have uniform properties. These lines can be shock waves, discretized expansion waves, or solid surfaces, and they are referred to as paths in the following description. As a result the output of the program is a list of polygons and corresponding lists that give the pressure, density, etc. in each polygon. This is very similar to the output of a two-dimensional finite-volume code except that the grid is very coarse in most of the flow. However, since the polygons are assembled during the flow computation, there is no need to assemble a grid $a$ priori.

In addition to the wave theory of Sec. II, models are constructed for regions of the flow in which the local Mach number is less than one. Because information can travel upstream in these regions, they are somewhat incompatible with the rest of the solution. The simple models presented here provide an architecture that allows them to be integrated with the otherwise supersonic flow.

\section{A. Description of the Proposed Algorithm}

The input to the proposed model consists of two parts. The first part determines the geometry of the flow, and it requires a list of polygons that specify the solid surfaces in the flow. For example the reference inlet shown in Fig. 2 has two input polygons: a list of several vertices for the scramjet vehicle body and a list of four vertices for the cowl. The second part of the input is the initial conditions. These are the conditions upstream of the input polygons. For example, in Fig. 3, the initial conditions specify the flow along the left edge of the image, which consists of the freestream conditions above and below the vehicle and the combustor exhaust conditions between the body and the cowl.

Once the flow geometry and initial conditions have been specified, the program proceeds by marching downstream and searching for wave interactions or vertices of the input geometry. In order to accomplish this, the program begins its analysis at the farthest upstream $x$-coordinate, $x_{\min }$, and proceeds downstream toward the right-hand $x$-coordinate, $x_{\max }$. As the program proceeds downstream, it tracks the positions of all the straight lines in the flow, which includes waves, the surfaces of the input geometry, and the boundaries of the flow domain. Within the program each of these straight lines is tracked as a path that consists of a point and a propagation angle. The program keeps track of all of the paths that intersect the vertical line corresponding to the current $x$-coordinate.

With the set of paths at a given $x$-coordinate given, a list of possible intersection points can be determined. Suppose the current $x$-coordinate is $x_{k}$, and the paths are listed by ascending $z$-coordinates. Then two paths with coordinates $z_{j}$ and $z_{j+1}$ have a downstream intersection point of

$$
x_{k, j}=x_{k}+\frac{y_{j+1}-y_{j}}{\tan \sigma_{j}-\tan \sigma_{j+1}}
$$

provided that $\sigma_{j}<\sigma_{j+1}$. Then the $x$-coordinate of the next interaction point, $x_{k+1}$ is the minimum of all the $x_{k, j}$ and all of the vertices of the input polygons that are downstream of $x_{k}$. 
Once an interaction point is found, a three-step process takes place. First, the nature of the interaction is determined, and the local flow problem is solved. This consists of determining the state (pressure, flowpath angle, etc.) above and below the interaction point. If one of the paths is a surface boundary, the downstream conditions are determined by either a shock or an expansion. Otherwise, the solution is determined by a Riemann problem as described in Sec. II.D. The second step is to add the coordinates of the interaction point to the polygons of the interacting regions. Finally, the list of waves and their propagation angles at the current $x$-coordinate are updated so that the coordinates of the next interaction point can be determined.

\section{B. Nozzle geometry}

For validation purposes the proposed method is applied to the nozzle geometry listed in Table 1. This is the nozzle pictured in Figs. 2 and 8.

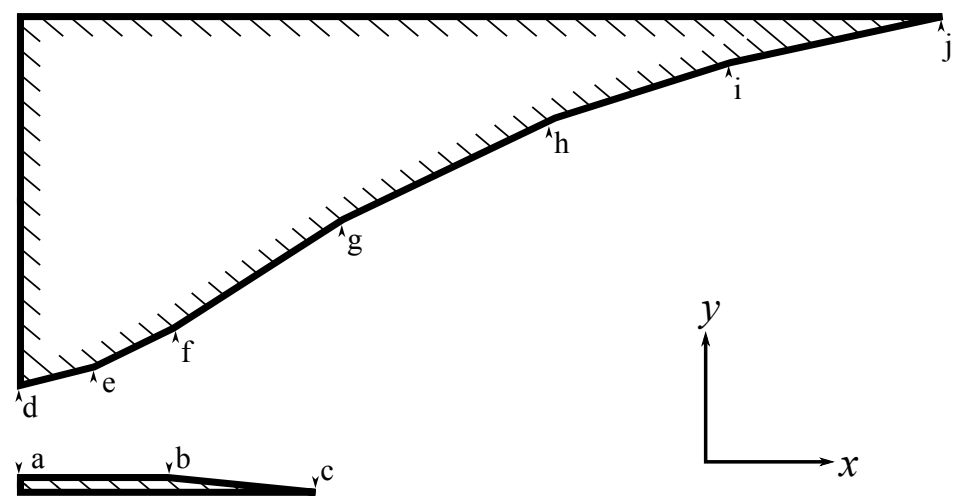

Figure 8. Geometry of example geometry with labels at key vertices

Table 1. Scaled geometry of example nozzle. The physical scales are set by $L_{5}=5 \mathrm{~m}$. Points a-c are the vertices of the cowl, and the remaining points give the locations of the vertices of the main vehicle body.

\begin{tabular}{ccc}
\hline \hline$k$ & $\left(x_{5 k}-x_{5 \mathrm{a}}\right) / L_{5}$ & $\left(y_{5 \mathrm{k}}-y_{5 a}\right) / L_{5}$ \\
\hline $\mathrm{a}$ & 0.000 & 0.000 \\
$\mathrm{~b}$ & 0.160 & 0.000 \\
$\mathrm{c}$ & 0.320 & -0.016 \\
\hline $\mathrm{d}$ & 0.000 & 0.100 \\
$\mathrm{e}$ & 0.080 & 0.120 \\
$\mathrm{f}$ & 0.166 & 0.162 \\
$\mathrm{~g}$ & 0.350 & 0.280 \\
$\mathrm{~h}$ & 0.580 & 0.390 \\
$\mathrm{i}$ & 0.770 & 0.450 \\
$\mathrm{j}$ & 1.000 & 0.500 \\
\hline \hline
\end{tabular}

\section{Boundary Conditions}

We selected a combination of freestream conditions and combustor conditions that would highlight the role of recombination in the nozzle. The conditions, which correspond to flight at an altitude of $45 \mathrm{~km}$ and a Mach number of 18.0, are shown in Table 2. The molecular composition corresponds to stoichiometric combustion of $\mathrm{H}_{2}$ and a dissociation 
fraction of $10 \%$. In this case, that means that $10 \%$ of the mass of hydrogen and oxygen atoms are in the form of radicals $(\mathrm{H}$ and $\mathrm{OH})$, and the remaining mass is $\mathrm{H}_{2} \mathrm{O}$. The nitrogen and argon atoms are left in their freestream state.

Table 2. Boundary conditions for nozzle validation case. The post-combustor state corresponds to the condition between the vehicle body and cowl, and the freestream condition is used as the boundary condition above and below the vehicle.

\begin{tabular}{ccc}
\hline \hline Property & Freestream & Post-combustor \\
\hline$\rho$ & $0.001966 \mathrm{~kg} / \mathrm{m}^{3}$ & $0.002240 \mathrm{~kg} / \mathrm{m}^{3}$ \\
$p$ & $149.1 \mathrm{~Pa}$ & $1094 \mathrm{~Pa}$ \\
$T$ & $264.2 \mathrm{~K}$ & $1400 \mathrm{~K}$ \\
$u$ & $5865 \mathrm{~m} / \mathrm{s}$ & $5131 \mathrm{~m} / \mathrm{s}$ \\
$Y_{\mathrm{N}_{2}}$ & 0.755590 & 0.734173 \\
$Y_{\mathrm{O}_{2}}$ & 0.231522 & 0 \\
$Y_{\mathrm{Ar}}$ & 0.012888 & 0.012523 \\
$Y_{\mathrm{H}_{2} \mathrm{O}}$ & 0 & 0.227974 \\
$Y_{\mathrm{H}}$ & 0 & 0.001417 \\
$Y_{\mathrm{OH}}$ & 0 & 0.023913 \\
\hline \hline
\end{tabular}

\section{Other Models Used for Comparison}

In addition to the proposed two-dimensional ROM, several other models were considered. The first of these is simply the proposed ROM itself but without finite-rate chemistry. In that model, the chemistry mechanism has been replaced with frozen chemistry, which gives some insight into how significant the chemistry actually is in the scramjet nozzle. The remaining two models consist of one with higher fidelity (CFD) and one with lower fidelity (an attempt at a one-dimensional model).

\section{A. High-fidelity CFD Solution}

We used the commercial CFD package CFD++ ${ }^{16}$ to obtain a high-fidelity analysis of the same two-dimensional nozzle geometry with the same boundary conditions given in Fig. 8 and Tables 1 and 2. The CFD solution included viscosity, compressibility, and finite-rate chemistry. The turbulence in the flow was modeled using a standard $k-\varepsilon$ scheme. The two-dimensional grid consisted of 300 thousand cells with a higher density of cells in the boundary layers and along the slip lines downstream of the trailing edges. For the scramjet vehicle surfaces, we used a fixed-temperature boundary coundition with $T_{\text {wall }}=1000 \mathrm{~K}$.

To obtain a better comparison of the computational times required for each model, we completed the CFD analysis on the same computer as the reduced-order models. The computer in question has a $2.66 \mathrm{GHz}$ processor with $3 \mathrm{~GB}$ of RAM. The CFD computation required approximately 8 hours for the normalized residuals to converge to $10^{-4}$ while the reduced-order models required at most a few seconds.

\section{B. Quasi-one-dimensional Model}

To demonstrate the two-dimensional nature of the nozzle flow, a model that is as close as possible to one-dimensional was constructed. A true one-dimensional model is not possible because of the free boundary of the exhaust plume that starts at the trailing edge of the cowl. The location of the exhaust plume boundary has a significant impact on the thrust, and it cannot be calculated without considering wave interactions.

To resolve this problem, we use the proposed two-dimensional model, only with frozen chemistry, to determine the exhaust plume boundary. Once the geometry of the plume has been determined, we apply a one-dimensional model with variable area. Although this method is thus dependent on the two-dimensional model, it offers some insight because it separates the effects and computational difficulties associated with two-dimensional flow and finite-rate chemistry.

The primary purpose of the quasi-one-dimensional model is to provide an alternate method to analyze the finite-rate chemistry. The wave-interaction model has been verified for two-dimensional flows without chemistry ${ }^{9}$ and should provide a good estimate of the exhaust plume boundary. However, incorporating chemistry into the two-dimensional 
model is the subject of this paper, and the quasi-one-dimensional model provides the simplest basis for comparison we could configure. This model would be less useful if the chemistry had a large effect on the position of the exhaust plume boundary, but that turns out not to be the case.

The equations of motion for this quasi-one-dimensional model are exactly those in Sec. II.B. The distance variable, $\xi$, is simply the horizontal coordinate, $x$. The area at a given $x$-coordinate is known because the exhaust plume boundary is known, so $d A / d \xi$ can be calculated at any point.

\section{Results}

The detailed analysis includes four models, which are summarized in Table . In addition to the two models described in the previous two subsections, the reduced-order two-dimensional model is used with two chemical mechanisms. The first mechanism is the Jachimowsk ${ }^{14}$ algorithm, and the second mechanism is simply frozen gas. The primary difference between the high-fidelity (CFD) model and the others is the presence of viscous boundary layers. All of the reduced-order models are inviscid. This combination of four models gives an opportunity to isolate the effects of chemistry, viscosity, and two-dimensionality.

Table 3. List of four models and the sections giving their descriptions

\begin{tabular}{ll}
\hline \hline Model & Section \\
\hline Proposed 2D ROM with finite-rate chemistry & Section III \\
Proposed 2D ROM with frozen chemistry & Section III \\
CFD++ with finite-rate chemistry & Section IVA \\
Quasi-1D model & Section IVB \\
\hline \hline
\end{tabular}

To compare all four models directly, the two-dimensional results must be collapsed into one-dimensional analysis. To do this, we averaged the two-dimensional results along vertical lines using the mass flux $(\rho u)$ as weighting. These one-dimensional results are shown in Figs. 9-11. Figure 9a shows that all three two-dimensional models give a very similar result for pressure, while the one-dimensional model gives a significantly different result. Already this indicates that the two-dimensional nature of the supersonic expansion fans is very important in the nozzle flowpath. It also gives an early indication that chemistry does not play a significant role, while it is inconclusive on viscosity since pressure is approximately constant across a boundary layer.

Figures 9b-c, which show the averaged temperature and velocity, respectively, add the conclusion that the boundary layer plays an important role. The error of approximately $8 \%$ in the exit velocity comes mostly from the fact there is a portion of the flow near the wall where the flow velocity is close to zero. This low velocity is also the reason for the higher temperature in the CFD result, as some of the gas's kinetic energy is converted to sensible enthalpy in the low-speed regions. Comparing the two two-dimensional ROMs in Figs. 9b-c is more difficult. The temperature plot shows that the chemistry has a noticeable effect on the sensible enthalpy, but the kinetic energy is so high that the additional thermal energy has only a small effect on the velocity. Moreover, the velocity plot shows that despite having less available energy, the model with frozen chemistry actually predicts a slightly higher exit velocity. This is due to the tendency of heat addition to drive a flow toward Mach 1, and the result would be reversed in a longer nozzle with a higher expansion ratio.

At this point we should note that the nozzle and boundary conditions in this paper were selected to exaggerate the effect of finite-rate chemistry. With a lower initial velocity, the radical species recombine in so quickly that the vast majority of the nozzle flow is in chemical equilibrium. Although various conclusions could be drawn from this, the primary reason we selected these conditions is to demonstrate the ability of this model to accurately predict the reactions in the nozzle flow.

Figures 10 and 11 show the one-dimensional averaged mass fractions for several key species. The first of these plots, Fig. 10a shows that most of the $\mathrm{OH}$ recombines to form $\mathrm{H}_{2} \mathrm{O}$, but in the CFD results, another chemical pathway becomes active, and some of the $\mathrm{H}_{2} \mathrm{O}$ atoms dissociate. Most of the dissociated $\mathrm{H}_{2} \mathrm{O}$ appears to go into $\mathrm{O}$ radicals. Considering that both of the reduced-order models with chemistry make similar predictions, this suggests that the boundary layer also plays an important role (directly or indirectly) in the chemistry. $\mathrm{The}_{2} \mathrm{H}_{2}$ plot, which is shown in Fig. 10b, shows that the three chemistry models predict a similar recombination profile, although the mass fraction is very small.

The results for the radical species, shown in Fig. 11, are similar for all three models with finite-rate chemistry. The 


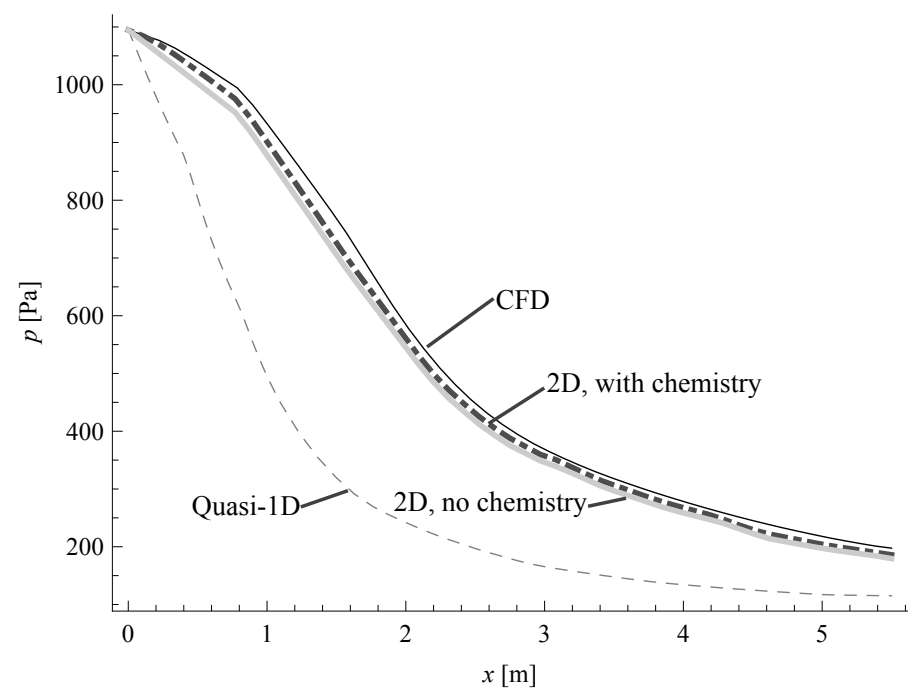

a) Pressure

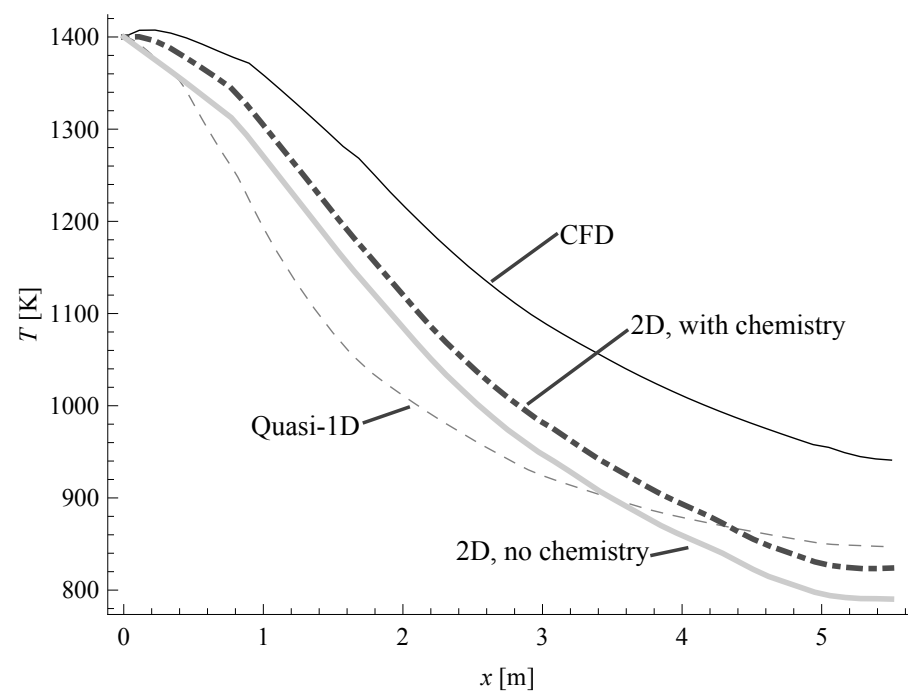

b) Temperature

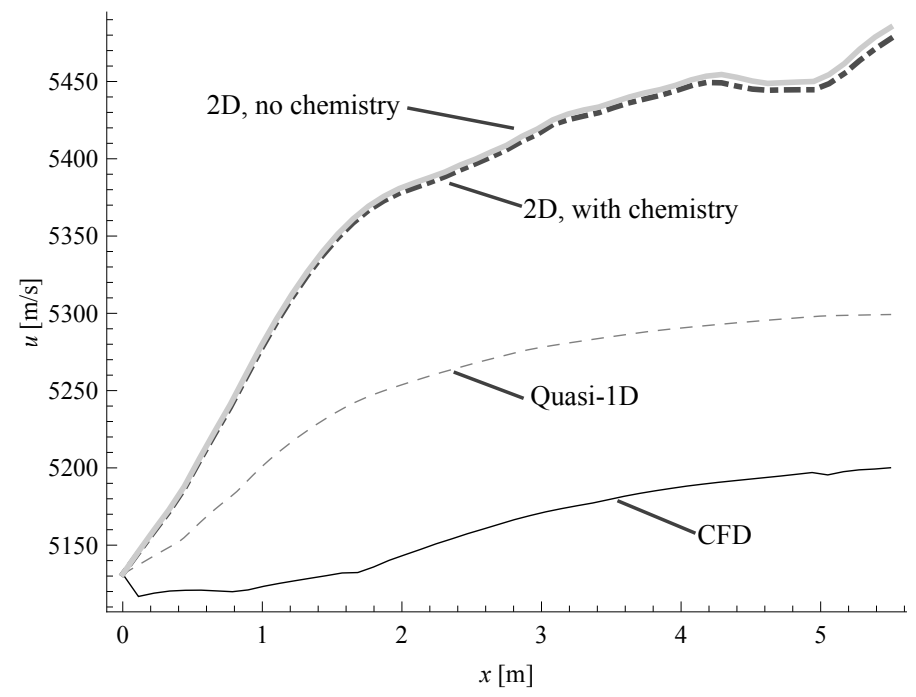

c) Velocity

Figure 9. Plots of mass flow-averaged quantities versus $x$ for various models. 
plot of $\mathrm{H}$ recombination in Fig. 11b seems to show more recombination in the CFD model, but a closer look at the vertical axis shows that all three models predict a fairly low amount of recombination.

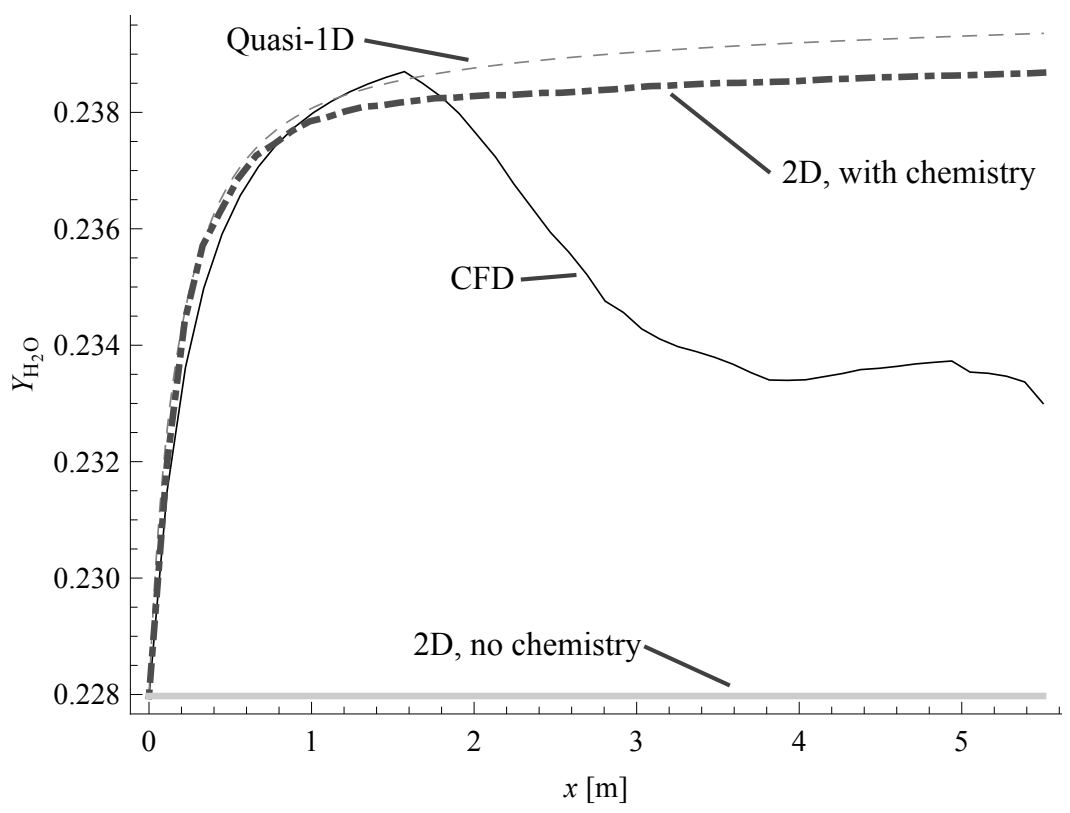

a) $Y_{\mathrm{H}_{2} \mathrm{O}}$

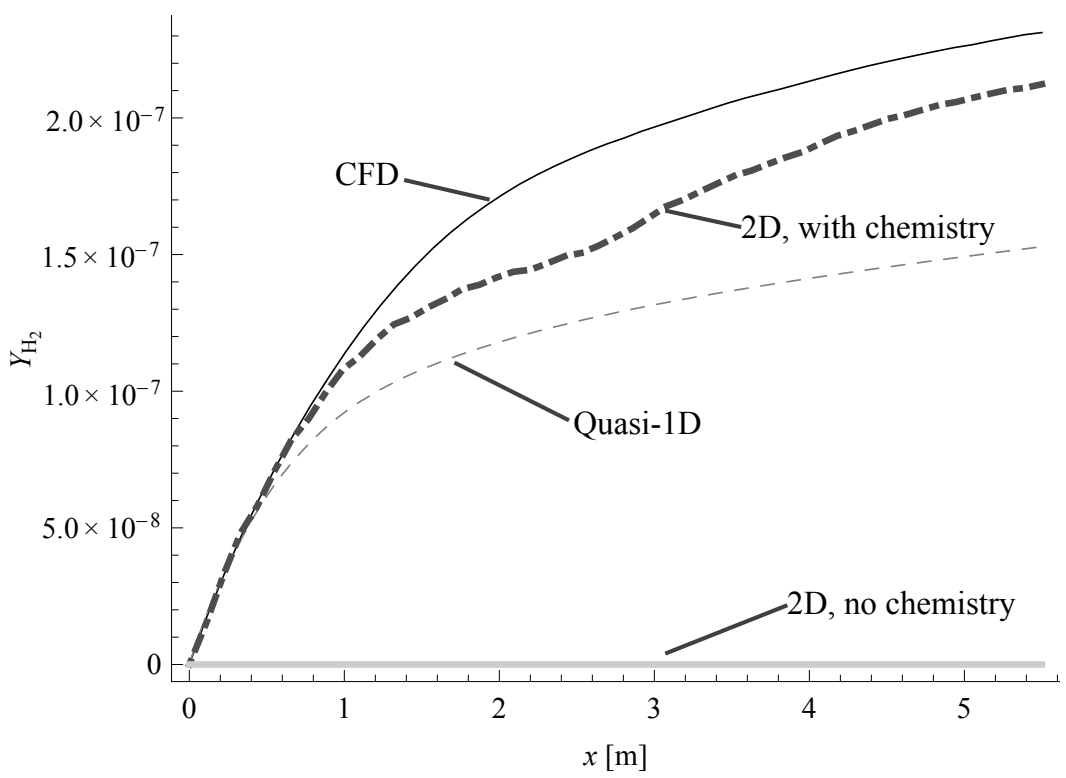

b) $Y_{\mathrm{H}_{2}}$

Figure 10. Plots of mass flow-averaged stable species mass fractions versus $x$ for various models.

Figure 12 shows the boundaries of the exhaust plume for the two-dimensional models. A significant portion of the boundary is just the vehicle itself, and the remaining portion of the boundary is determined by the streamlines starting at the two trailing edges. Also, note that the plume from the reduced-order model with frozen chemistry is the one used in the quasi-one-dimensional model. The graph shows that all three models predict a very similar plume. Clearly neither viscosity nor chemistry have a qualitative effect on the shape of the exhaust plume.

The higher-temperature and lower-velocity regions near the vehicle wall seem to be the main cause of the differences among the models. To show this result in more detail, Figs. 13-15 show a two-dimensional comparison of the CFD results with the results of the full reduced-order model. Figure 13 shows that both models predict a similar pattern for $\mathrm{OH}$ recombination except near the wall. This result verifies the capability of the proposed reduced-order 


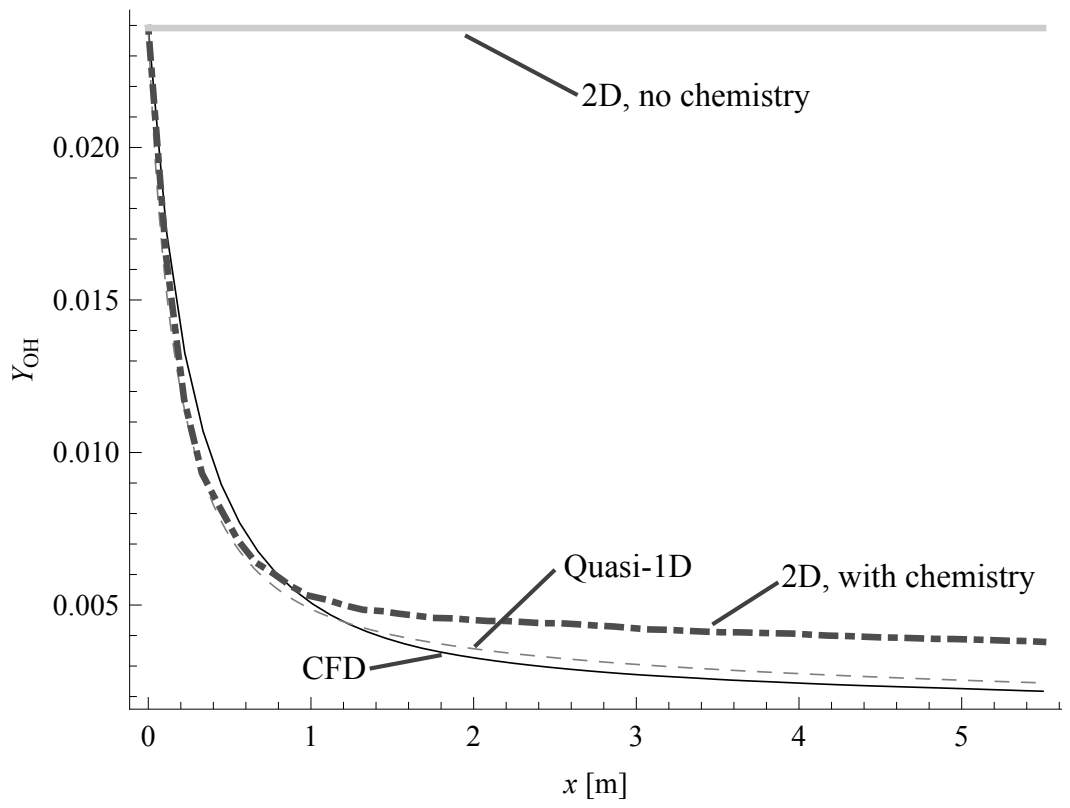

a) $Y_{\mathrm{OH}}$

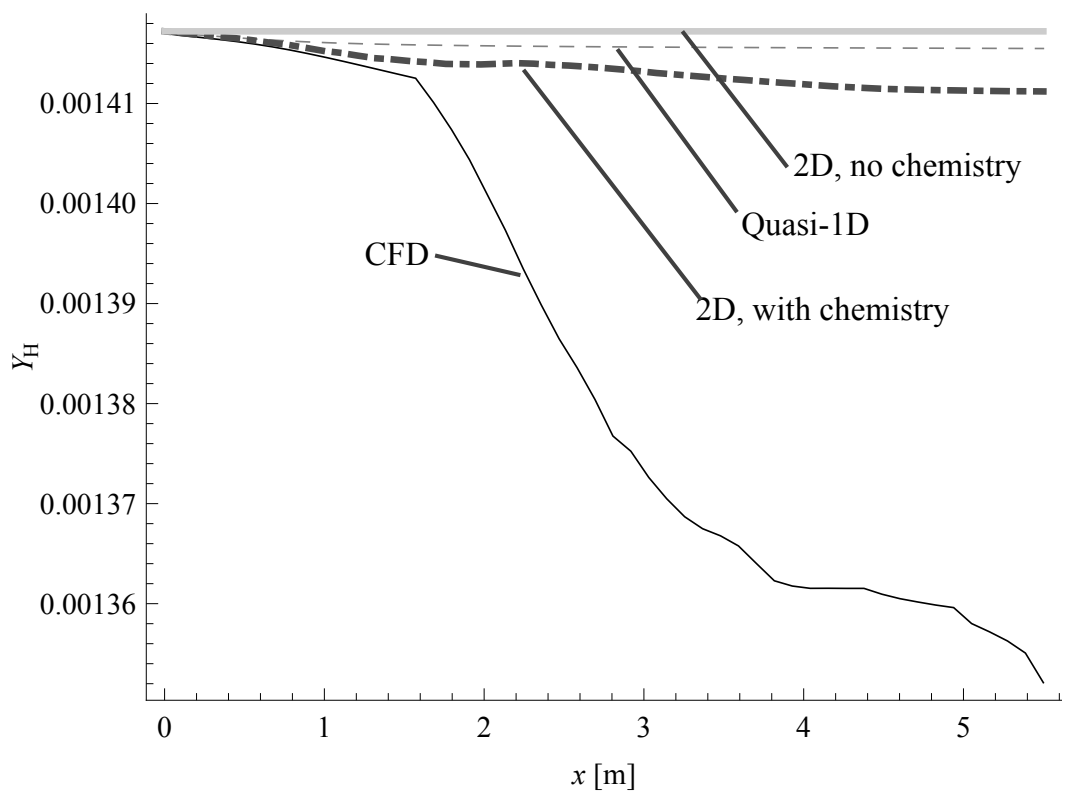

b) $Y_{\mathrm{H}}$

Figure 11. Plots of mass flow-averaged radical species mass fractions versus $x$ for various models. 


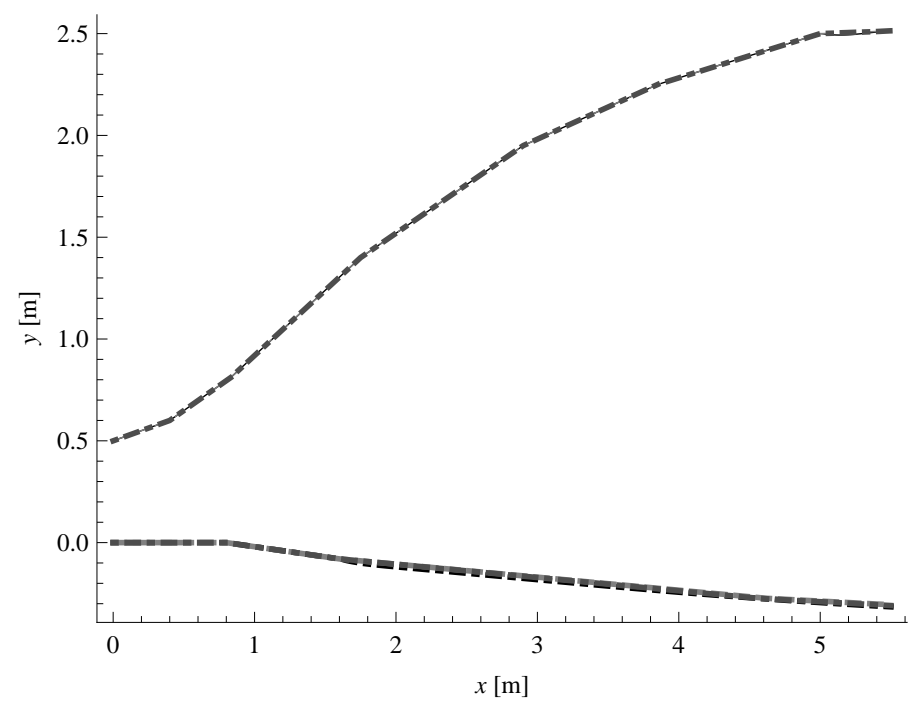

Figure 12. Graph of exhaust plume boundary using the various models

model to capture supersonic flow phenomena and finite-rate chemistry. It should also be noted that all of the other species relevant to the exhaust plume have two-dimensional mass fraction plots that are very similar to those in Fig. 13 or very similar to the reverse of those in Fig. 13.

Combining the results shown in Fig. 13-15 confirms that more $\mathrm{OH}$ recombination occurs in the high-temperature and low-speed region than the inviscid model is able to predict. The higher temperature increases the reaction rate, and the lower flowspeed gives the chemistry more time to take its course.

\section{Conclusions}

A reduced-order two-dimensional model was developed that can analyze shock waves, expansion fans, and finiterate chemistry. The model was found to be particularly accurate in determining the boundary of the exhaust plume, which is essential to thrust calculations. Recombination can also be modeled as long as the flow is well-mixed before reaching the nozzle. However, the importance of recombination to thrust calculations was debatable, even for a set of conditions specifically selected to emphasize the importance of recombination.

The model does not have the capability to analyze boundary layers, which were found to play an important role. The boundary layer had a noticeable effect on all quantities except for pressure. These results make a strong case that a boundary layer model must be added to the reduced-order model.

\section{Acknowledgements}

This research was supported by AFRL grant FA 8650-07-2-3744 for the MACCCS (Michigan AFRL Collaborative Center for Control Science).

\section{References}

${ }^{1}$ Torrez, S. M., Driscoll, J. F., Dalle, D. J., Bolender, M. A., and Doman, D. B., "Hypersonic Vehicle Thrust Sensitivity to Angle of Attack and Mach Number," AIAA Atmospheric Flight Mechanics Conference, 2009, AIAA Paper No. 2009-6152.

${ }^{2}$ Torrez, S. M., Driscoll, J. F., Dalle, D. J., and Micka, D. J., "Scramjet Engine Model MASIV: Role of Mixing, Chemistry and Wave Interaction," 45th AIAA/ASME/SAE/ASEE Joint Propulsion Conference and Exhibit, 2009, AIAA Paper No. 2009-4939.

${ }^{3}$ Torrez, S. M., Driscoll, J. F., Dalle, D. J., and Fotia, M. L., "Preliminary Design Methodology for Hypersonic Engine Flowpaths," 16th AIAA/DLR/DGLR International Space Planes and Hypersonic Systems and Technologies Conference, 2009.

${ }^{4}$ Frendreis, S. G. V., Skujins, T., and Cesnik, C. E. S., "Six-Degree-of-Freedom Simulation of Hypersonic Vehicles," AIAA Atmospheric Flight Mechanics Conference, 2009, AIAA Paper 2009-5601.

${ }^{5}$ Bolender, M. A. and Doman, D. B., "Nonlinear Longitudinal Dynamical Model of an Air-Breathing Hypersonic Vehicle," Journal of Spacecraft and Rockets, Vol. 44, No. 2, 2007, pp. 374-387.

${ }^{6}$ Cubbage, J., N. A. Talcott, J., and Hunt, J. L., "Scramjet Exhaust Simulation Technique for Hypersonic Aircraft Nozzle Design and Aero- 


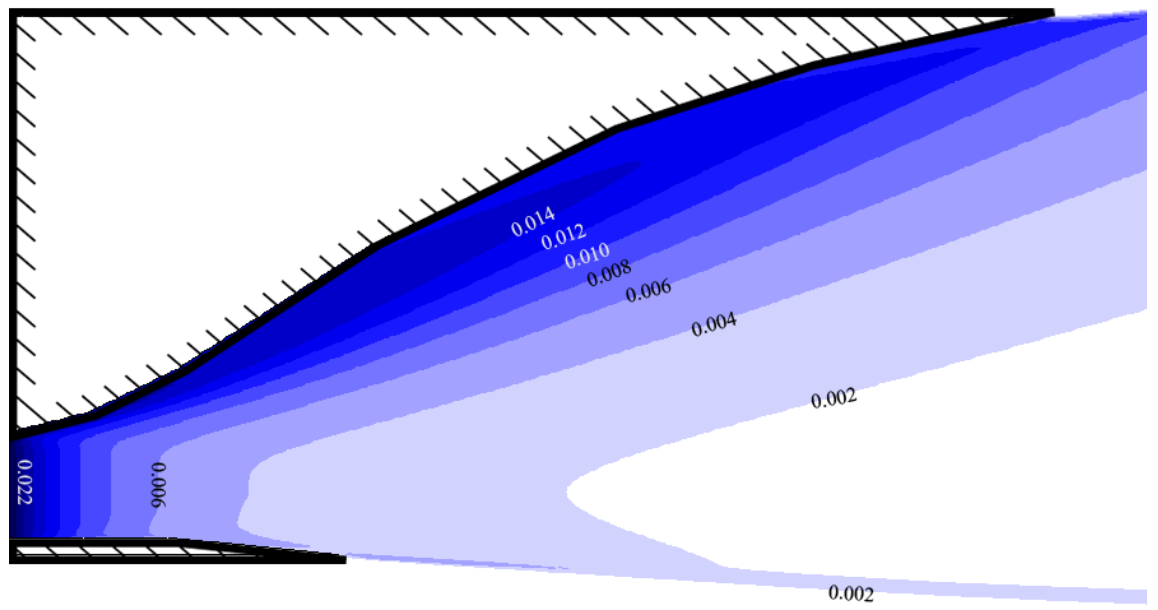

a) CFD++ solution

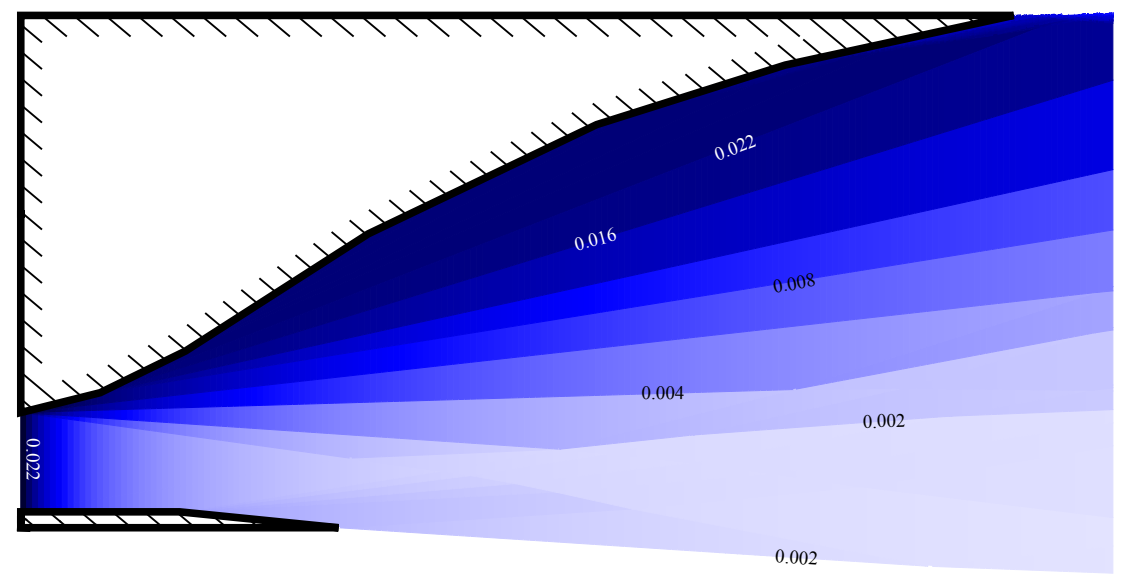

b) Proposed reduced-order model

Figure 13. Contours of $\mathrm{OH}$ mass fraction for two models. In both cases darker shades of blue represent higher mass fractions with a maximum of 0.024 . 


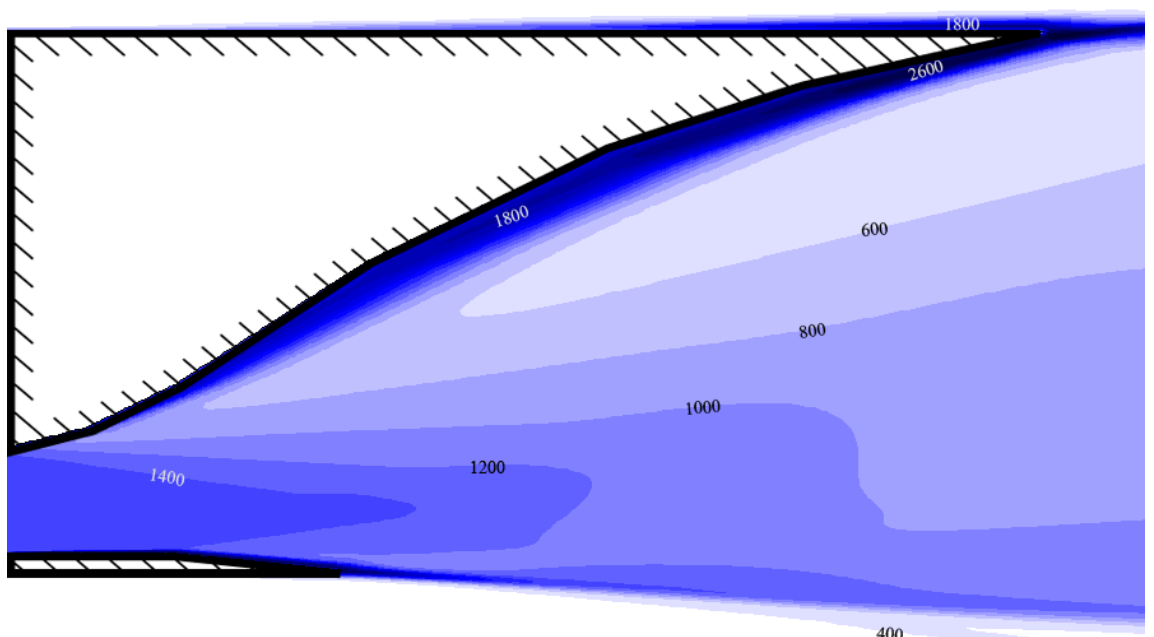

400

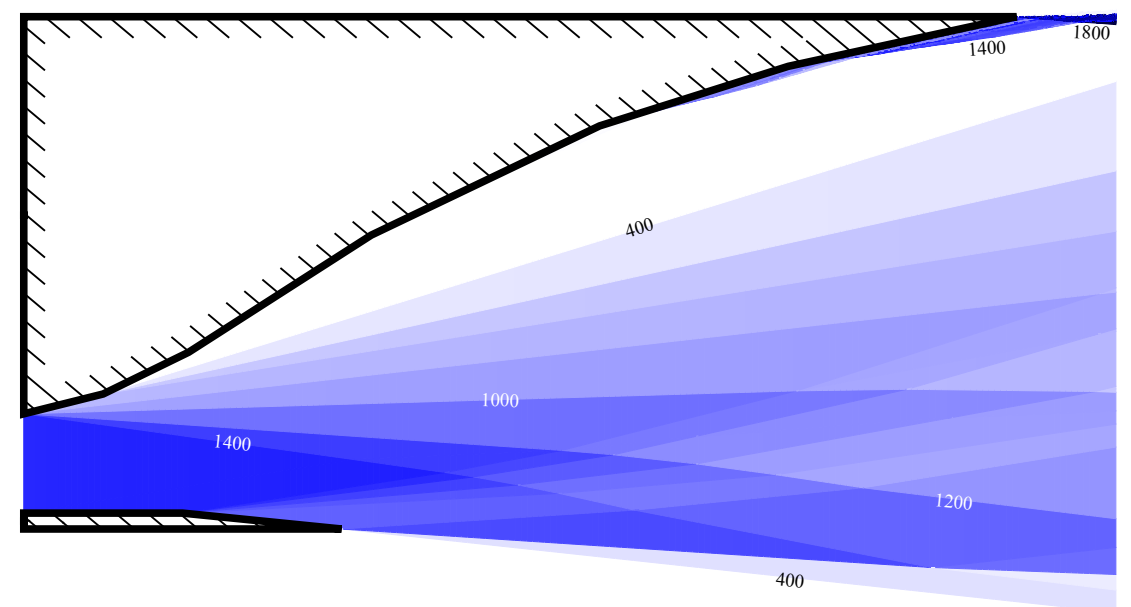

b) Proposed reduced-order model

Figure 14. Contours of temperature for two models. In both cases darker shades of blue represent higher temperatures with a maximum of $2600 \mathrm{~K}$. 


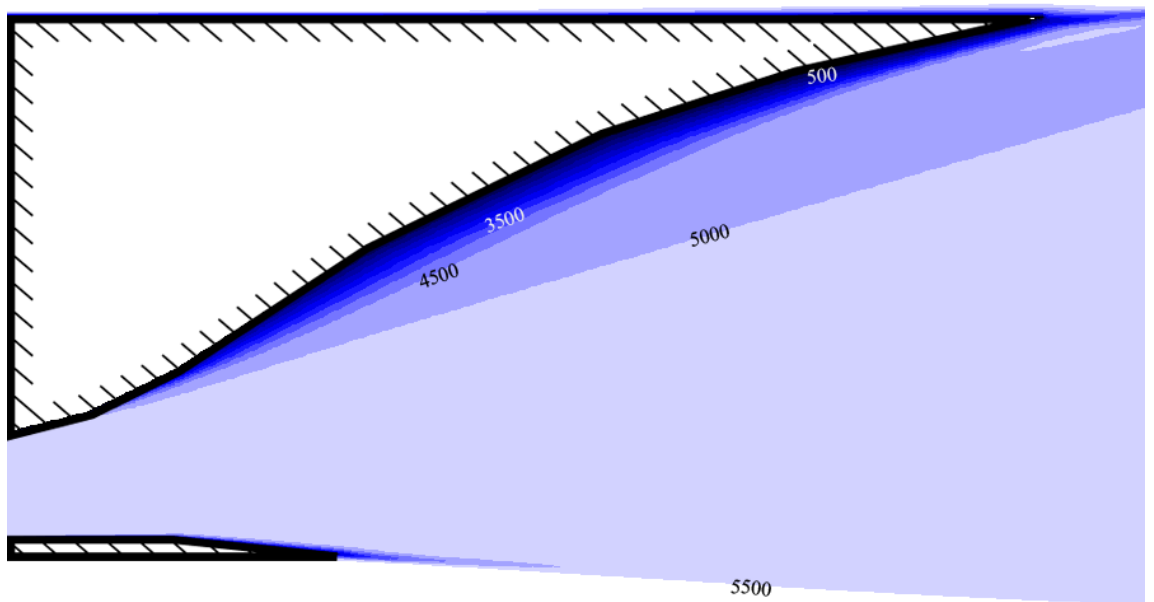

a) CFD++ solution

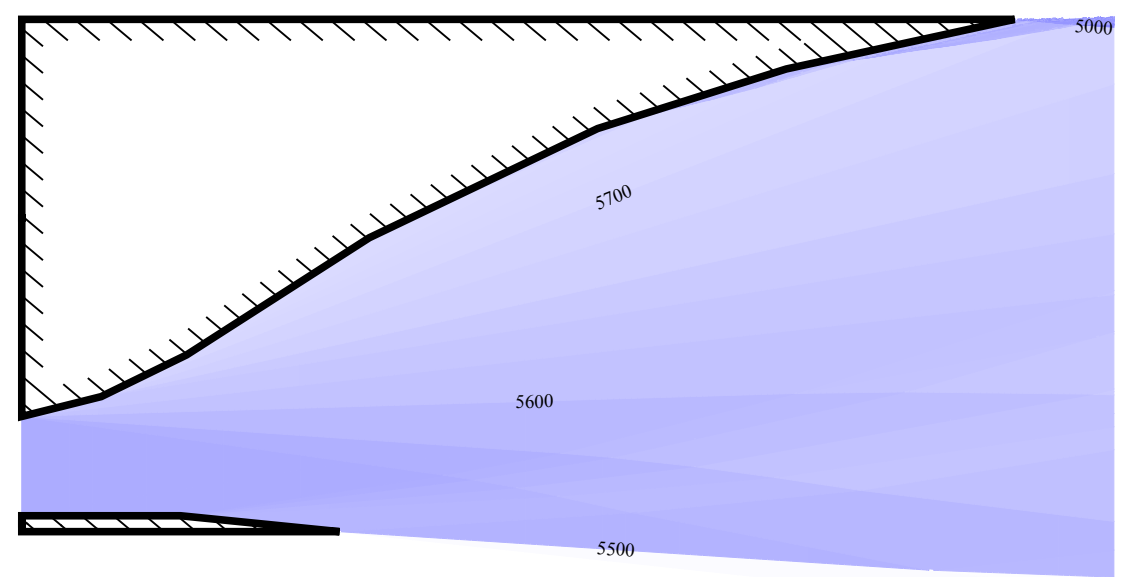

b) Proposed reduced-order model

Figure 15. Contours of temperature for two models. In both cases darker shades of blue represent lower flow speeds with a minimum of $0 \mathrm{~m} / \mathrm{s}$. White corresponds to the freestream velocity. 
dynamic Tests," 15th AIAA Aerospace Sciences Meeting, 1977.

${ }^{7}$ Ishiguro, T., Takaki, R., Mitani, T., and Hiraiwa, T., "Three-Dimensional Analysis of Scramjet Nozzle Flows," Journal of Propulsion and Power, Vol. 10, No. 4, 1994, pp. 540-545.

${ }^{8}$ Sindir, M. M. and Lynch, D. E., "Overview of the State-of-the Practice of Computation Fluid Dynamics in Advanced Propulsion System Design,” 28th Fluid Dynamics Conference, 1997, AIAA Paper 1997-2124.

${ }^{9}$ Dalle, D. J., Fotia, M. L., and Driscoll, J. F., "Reduced-Order Modeling of Two-Dimensional Supersonic Flows with Applications to Scramjet Inlets," Journal of Propulsion and Power, Vol. 26, No. 3, 2010, pp. 545-555.

${ }^{10}$ Heiser, W. H. and Pratt, D. T., Hypersonic Airbreathing Propulsion, AIAA Pub., Washington, DC, 1994.

${ }^{11}$ Korte, J. J., "Parametric Model of an Aerospike Rocket Engine," 38th Aerospace Sciences, 2000, AIAA Paper 2000-1044.

${ }^{12}$ Korte, J. J., Salas, A. O., Dunn, H. J., Alexandrov, N. M., Follett, W. W., Orient, G. E., and Hadid, A. H., "Multidisciplinary Approach to Linear Aerospike Nozzle Design," Journal of Propulsion and Power, Vol. 17, No. 1, 2001, pp. 93-98.

${ }^{13}$ Ito, T. and Fujii, K., "Flow Field and Performance Analysis of the Simplified Clustered Aerospike Nozzles," 31st AIAA Fluid Dynamics Conference \& Exhibit, 2001, AIAA Paper 2001-2861.

14 Jachimowski, C. J., "An analytic study of the Hydrogen-Air reaction mechanism with application to SCRAMJET combustion," Technical Paper 2791, NASA, 1988.

${ }^{15}$ McBride, B. J., Zehe, M. J., and Gordon, S., "NASA Glenn Coefficients for Calculating Thermodynamic Properties of Individual Species," Tech. rep., NASA, September 2001, TP-2002-211556.

${ }^{16}$ Anon., “CFD++ User Manual,” Tech. rep., Metacomp Technologies, Inc., Agoura Hills, California, 2006. 